\title{
Fall prevention behaviour after participation in the Stepping On program: a pre-post
} study

\section{Anne Tiedemann ${ }^{a, d}$, Kate Purcella, Lindy Clemson ${ }^{b}$, Stephen R Lord ${ }^{c}$ and Catherine Sherrington ${ }^{\mathrm{a}}$}

a Institute for Musculoskeletal Health, School of Public Health, Faculty of Medicine and Health, University of Sydney, NSW, Australia

b Sydney School of Health Sciences, Faculty of Medicine and Health, University of Sydney, NSW, Australia

c Neuroscience Research Australia, UNSW Sydney, Australia

${ }^{d}$ Corresponding author: anne.tiedemann@sydney.edu.au

\section{Article history}

Publication date: March 2021

Citation: Tiedemann A, Purcell K, Clemson L, Lord SR, Sherrington C. Fall prevention behaviour after participation in the Stepping On program: a prepost study. Public Health Res Pract. 2020;31(1):e30122004. First published: 22 April 2020. https://doi.org/10.17061/ phrp30122004

\section{Key points}

- The Stepping On fall prevention program was associated with a significant increase in participation in balance and strength exercise, and safe walking strategies

- Participant satisfaction with the Stepping On fall prevention program was very high

- The main motivators for, and barriers to, future uptake of structured exercise for participants not currently exercising included their own health, availability and access to local programs, and time available to take part

\section{Abstract}

Objective: The Stepping On program has been shown to prevent falls among community-dwelling people in a research setting and was implemented statewide by the New South Wales (NSW) Ministry of Health in 2008. This study measured ongoing fall prevention strategies and behaviours undertaken by Stepping On participants during the 6 months after program completion. Secondary objectives were to document participant satisfaction with the program, and to identify motivators for, and barriers to, fall prevention behaviour and uptake of the strategy.

Methods: We conducted a pre-post prospective study among Stepping On program participants, with 6-month follow-up. Participants commenced Stepping On in 2015 and 2016 in 15 Local Health Districts across NSW. A study-specific survey was completed at baseline and 6 months after completion of Stepping On. Measures were current self-reported fall prevention strategies and behaviours; the Falls Behavioural (FaB) Scale; the Incidental and Planned Exercise Questionnaire (IPEQ); and motivators for, and barriers to, uptake of fall prevention strategies and behaviours.

Results: Baseline questionnaires were completed by 458 participants (mean age 77; standard deviation [SD] 6.7; 76\% female). Both baseline and follow-up surveys were completed by 291 participants (64\%; mean age 78; SD 6.9; 76\% female). Program satisfaction was high - 251 participants (86\%) completed the whole program, 284 (98\%) said it increased their awareness of falls, and 284 (98\%) would recommend Stepping On to others. There were statistically significant increases in the proportion of participants who reported doing regular balance and strength exercise (74\% vs 24\%; $p<0.0001$ ), and using safe walking strategies (78\% vs 51\%; $p<0.0001)$ at follow-up compared with baseline. There was also a significant improvement in the FaB Scale, indicating less risk-taking behaviour (mean increase 0.15 out of $4 ; 95 \%$ confidence interval [Cl] 0.12, 0.19; $p<0.0001$ ), and an increase in IPEQ- 
reported structured exercise (mean increase 2.0 hours per week; 95\% Cl 1.6, 2.5; $p<0.0001)$. The main motivators for, and barriers to, uptake of structured exercise included participants' health, availability and access to local programs, and the amount of time available to take part.

Conclusion: This study demonstrates the appeal of the Stepping On program, and its positive impact on fall prevention behaviours among adults in the community aged 65 years and older. It is important to note the study limitations - namely, the self-reported nature of the measures used and the large amount of missing data.

\section{Introduction}

Falls can have a devastating impact on older people, often resulting in serious injury, loss of confidence, reduced mobility and decreased independence. ${ }^{1}$ Falls are also associated with a threefold increase in the risk of admission to residential aged care, even after adjusting for other risk factors. ${ }^{2}$ The social and economic burden of falls to health systems and broader society is increasing as a result of the ageing of the population.

Annual fall rates among community-dwelling people aged 65 years and older in Australia range from one in five $^{3}$ to one in three people. ${ }^{4}$ Each year, falls lead to more than 92000 hospitalisations ${ }^{5}$ and more than 3200 deaths among Australians aged 65 years and older. The rate of fall-related hospitalisations for people aged 65 years and older has been increasing since 2002-03. ${ }^{3}$

Exercise is the single most effective intervention for preventing falls ${ }^{6}$ in older age. There is also evidence for benefits from other interventions, used alone or in combination, such as interventions to improve the safety of the home environment, visual interventions such as cataract surgery, and medication review. ${ }^{7}$

One example of an effective fall prevention program that combines exercise and group-based discussion of other risk factors is the Stepping On program ${ }^{8}$, developed and widely implemented in Australia. Stepping On aims to improve fall self-efficacy and encourage behavioural change to reduce falls. It focuses on improving lowerlimb balance and strength, improving home safety, teaching strategies and behaviours that promote safe mobility, encouraging regular visual screening, making adaptations to low vision, and encouraging medication review. Stepping On includes weekly 2-hour sessions conducted for 7 weeks, with a follow-up booster visit at week 12.

In the original trial, the Stepping On program was associated with a $31 \%$ reduction in fall risk among community-dwelling people aged 70 years and older who had fallen in the past year or were concerned about falling. ${ }^{8}$ The New South Wales (NSW) Ministry of Health has funded the implementation of the Stepping On program across NSW Local Health Districts (LHDs) as a key fall prevention initiative since 2008, and the Wisconsin
Institute of Healthy Ageing has provided training in the program across the US since 2006. ${ }^{9}$ Although there has been some evaluation of participant outcomes in practice settings ${ }^{10}$, there has been little evaluation in terms of ongoing behaviour change among program participants. The only study to date conducted a brief knowledge survey pre-program and 3 months post-program; it used a brief yes/no survey regarding behavioural steps linked to fall prevention at the 3-month follow-up. ${ }^{11}$

The primary objective of this study was to measure the impact of the Stepping On program by documenting fall prevention strategies and behaviours undertaken by program participants during the 6 months after they completed the Stepping On program. Secondary objectives were to document participant satisfaction with the program, and to identify motivators for, and barriers to, uptake of fall prevention behaviour and strategies.

\section{Methods}

A pre-post intervention prospective study was undertaken among Stepping On program participants. The study design and procedures were approved by the Human Research Ethics Committee at South Eastern Sydney LHD (approval number 14/141). Site-specific approval was granted by each of the 14 other LHDs involved. The study is reported with reference to the STROBE checklist for cohort studies. ${ }^{12}$

Potential participants were community-dwelling people aged 65 years and older, enrolled in the Stepping On program in 15 LHDs across NSW between 2015 and 2016. Stepping On program eligibility criteria are that the participant is aged 65 years or older, is cognitively intact, has experienced a fall in the past year or is fearful of falling. The only exclusion criterion for the current study was not having sufficient English to provide informed consent and to complete the study surveys; this was because of insufficient funds for language translation. We aimed to recruit 500 participants in total and to recruit from as many different LHDs as possible to maximise the diversity of participants included. 
Stepping On group facilitators introduced potential participants to the research study during the first, second or third Stepping On program session. They distributed study packs, which contained the participant information statement, the consent form, the baseline survey and a reply-paid envelope, to interested people. Research staff were available via telephone to answer questions about the study or to assist with completion of the survey, if needed. Study participation was voluntary and open to all eligible attendees of all Stepping On groups run during the recruitment period. People who chose not to participate were not disadvantaged in any way.

After giving written informed consent, participants completed a paper-based baseline survey, which took approximately 30 minutes, and returned the consent form and survey in a reply-paid envelope to the research team. A follow-up survey was posted or emailed to participants 6 months after they completed the Stepping On program. The study flow and procedures are illustrated in Figure 1. The baseline and follow-up surveys are included as supplementary files (available from: hdl.handle. net/2123/21974)

The baseline survey collected information on demographics, medical conditions, medications and fall history. Current fall prevention behaviours were assessed with a study-specific list of possible behaviours consistent with the behaviours discussed during the Stepping On program. Examples include the use of safe walking strategies - these are practical strategies for safe mobility, (e.g. 'scanning ahead' for hazards, or safety tips for negotiating stairs), or the use of environmental lighting (e.g. sensor-activated night lights). Current behaviours were also assessed with the Falls Behavioural (FaB) Scale ${ }^{13,14}$, a reliable and valid tool for measuring fall prevention behaviours that is sensitive to change in behaviour following interventions for fall prevention. 13,14 $^{1}$ The FaB Scale consists of 30 statements describing everyday behaviours and actions (e.g. 'I hurry to answer the phone'), and the respondent is required to select how often they do each activity, on a four-point scale ranging from 'never' to 'always'.

The baseline survey also included an assessment of current physical activity, measured using the Incidental and Planned Exercise Questionnaire (IPEQ). ${ }^{15}$ The IPEQ consists of 10 questions that measure the frequency and duration of both planned and incidental physical activity and exercise. It is validated for use with older people. The IPEQ allows calculation of a total activity score, as well as subscores that measure the number of hours per week spent in incidental activity, walking activity, planned activity, planned walking activity and planned sport activity. This study used the IPEQ (version W) to measure physical activity during the past week. ${ }^{15}$

At follow-up, participants again completed a written survey that included the FaB Scale and the IPEQ, as well as questions about their perceptions of the Stepping On program, strategies and behaviours they had adopted to reduce their risk of falls, and motivators for, and barriers to, the uptake of fall prevention strategies and behaviours.

Descriptive statistics were used to summarise the data. Changes in participation in evidence-based fall prevention interventions and behaviours among individuals between baseline and follow-up were compared using the McNemar test (a statistical test used on paired dichotomous data to see whether there has been a change in the proportions). Paired samples t-tests were used to compare the mean IPEQ and FaB scores between baseline and follow-up.

\section{Results}

Participants numbered 458 (346 females; 112 males) and had a mean age of 77 years (standard deviation [SD] 6.7). Of the total, 184 participants (40\%) lived alone, and most ( $n=446 ; 97 \%)$ spoke English at home. A total of 268 participants (59\%) had experienced at least one fall in the past year. Participants reported an average of 4.2 chronic medical conditions and undertook an average of 27.6 hours of incidental and planned physical activity per week. About two-thirds of participants ( $n=305 ; 67 \%)$ attended Stepping On groups in metropolitan LHDs; the remaining one-third ( $n=153$; 33\%) attended Stepping On programs in rural LHDs. The baseline characteristics of the study sample are shown in Table 1.

Figure 1. Participant flow through the study

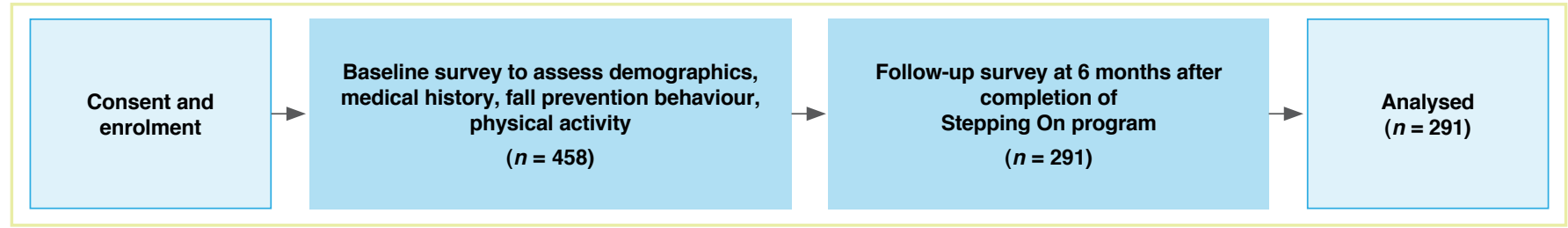


Table 1. Baseline characteristics of study participants $(n=458)$

\begin{tabular}{lc}
\hline Characteristic & Value \\
\hline Age - mean (SD) & $77.2(6.7)$ \\
Female sex - number (\%) & $346(76)$ \\
Male sex - number (\%) & $112(24)$ \\
English spoken at home - number (\%) & $446(97)$ \\
Lives alone - number (\%) & $184(40)$ \\
Fallen in the past 12 months - number (\%) & $268(59)$ \\
Total medical conditions - mean (SD) & \\
Attended Stepping On program in & $4.2(2.4)$ \\
metropolitan Local Health District - & $325(66)$ \\
number (\%) & \\
IPEQ total physical activity (hours/week) - & $27.6(17.7)$ \\
mean (SD) & \\
FaB score - mean (SD) & \\
\hline
\end{tabular}

$\mathrm{FaB}=$ Falls Behavioural Scale; IPEQ = Incidental and Planned Exercise Questionnaire; SD = standard deviation

a Medical conditions were selected by participants from arthritis, osteoporosis, gastrointestinal disease, cognitive impairment, angina/heart disease, congestive heart disease, visual impairment, peripheral vascular disease, atrial fibrillation, high blood pressure, diabetes, asthma, stroke/TIA, depression, gout, anxiety/panic disorder, cancer, hearing impairment

b Scored out of 4

Most participants found out about the Stepping On program from a friend or family member $(n=119 ; 26 \%)$, a health professional other than a doctor $(n=115 ; 25 \%)$, or a doctor $(n=65 ; 14 \%)$. The most common reasons for joining the Stepping On program were to improve balance ( $n=185 ; 40 \%)$ or to prevent falling ( $n=113 ; 25 \%$ ).

The 6-month follow-up survey was completed by 291 participants (64\%; 220 females, 71 males; mean age 78; SD 6.9). Reasons for loss to follow-up included illness or death $(n=8)$, incorrect contact details $(n=4)$, withdrawal due to other reasons $(n=6)$ and nonreturn of survey $(n=149)$. Participants who withdrew before follow-up had similar baseline characteristics to the responders in terms of mean age (77 years vs 78 years; $p=0.13$ ), proportion of females ( $73 \%$ vs $76 \% ; p=0.4)$, proportion who had fallen in the past year ( $61 \%$ vs $57 \% ; p=0.4)$, mean number of medical conditions ( 4.4 vs $4.1 ; p=0.2$ ) and mean total weekly physical activity ( 26 hours vs 29 hours; $p=0.09$ ).

Most participants in the follow-up sample $(n=251$; $86 \%)$ completed the entire Stepping On program. The most common reasons for noncompletion included ill health $(n=24)$, transport difficulties $(n=4)$ and perception of low program usefulness $(n=4)$. Seventysix participants $(26 \%)$ reported falling at least once in the 6-month period after they completed the Stepping On program. The analyses comparing fall prevention behaviours and strategies before and after the Stepping
On program include only those participants who completed both surveys.

Overall, participant perceptions of the Stepping On program were very positive. Most participants $(n=284$; 98\%) believed that the Stepping On program increased their awareness of falls; most $(n=278 ; 96 \%)$ believed that it motivated them to improve their safety; and most $(n=190 ; 65 \%)$ felt more independent as a result of taking part in the program. Most participants $(n=285 ; 98 \%)$ stated that they would recommend the Stepping On program to family and friends.

\section{Uptake of behaviours and strategies to prevent falls}

Among the 291 participants who completed the follow-up, most were already using a range of strategies to prevent falls at baseline when they started the Stepping On program. The most common of these were checking the fit and slipperiness of shoes and slippers $(n=208 ; 72 \%)$, taking a vitamin D supplement ( $n=158 ; 54 \%$ ), using safe walking strategies ( $n=147 ; 51 \%)$ and changing the home environment to improve safety $(n=127 ; 44 \%)$. Only one-quarter of participants were regularly taking part in strength and balance exercises, and only $83(29 \%)$ had seen their doctor or pharmacist for medication review.

Six months after completing the Stepping On program, $74 \%(n=218)$ of respondents reported regularly taking part in strength and balance exercises, and $78 \%(n=226)$ reported using safe walking strategies. Significantly more individuals who were not undertaking these behaviours at baseline were doing so at followup. However, there was little change in the use of other fall prevention strategies and behaviours (Table 2). At follow-up, there was a significant decrease in the overall proportion of people who reported having cataract surgery (from $43 \%$ at baseline to $11 \%$ at follow-up); however, 15 people did report a new occurrence of having cataract surgery at follow-up. Hence, the overall difference in proportions likely reflects people already having had the procedure at baseline. Similarly, we identified a lower overall proportion of people who reported having taken a vitamin $\mathrm{D}$ supplement at followup (54\% at baseline and $30 \%$ at follow-up), despite 21 new people reporting using this strategy at follow-up but not at baseline.

\section{Participation in regular strength and balance exercise}

Of the 218 participants who were taking part in regular strength and balance exercise at follow-up, 112 (51\%) were already doing an exercise program before Stepping On and continued with it at least weekly, and 81 (37\%) commenced a new exercise program and participated at least weekly. Home-based exercise programs were the most commonly reported ( $n=115 ; 53 \%)$, with just over half of this group ( $n=64 ; 56 \%)$ continuing their Stepping 
Table 2. Strategies and behaviours used by participants to prevent falls at baseline and follow-up ( $n=291)$

\begin{tabular}{lccc}
\hline & \multicolumn{2}{c}{ Number (\%) of people } \\
\cline { 2 - 3 } Strategy & Baseline & Follow-up & $\begin{array}{c}\text { People who had started using } \\
\text { strategy at follow-up }^{\text {a }}\end{array}$ \\
\hline Regular participation in strength and balance exercises & $71(24)$ & $218(74)$ & $151(52) ; p<0.0001$ \\
Cataract surgery & $125(43)$ & $33(11)$ & $15(5) ; p<0.0001$ \\
Safe walking strategies & $147(51)$ & $226(78)$ & $105(36) ; p<0.0001$ \\
Improved lighting in the home & $65(22)$ & $62(21)$ & $29(10) ; p=0.80$ \\
Changed home environment to improve safety & $127(44)$ & $111(38)$ & $45(15) ; p=0.15$ \\
Medication review by GP or pharmacist & $83(29)$ & $69(24)$ & $32(11) ; p=0.14$ \\
Vitamin D supplement & $158(54)$ & $86(30)$ & $21(7) ; p<0.0001$ \\
Checked shoes and slippers to ensure safety & $208(71)$ & $215(74)$ & $54(19) ; p=0.55$ \\
\hline
\end{tabular}

a Statistical significance of change from baseline to follow-up was assessed using the McNemar test.

On exercises at home. For participants undertaking a group-based program, the most common programs were gentle exercise or exercise for seniors, Tai Chi, swimming or water-based exercises, and yoga. For those who reported not currently participating in an exercise program ( $n=73)$, almost one-third $(n=23 ; 32 \%)$ intended to start one soon, while $41(56 \%)$ had no intention of starting an exercise program in the near future.

Participants who were not taking part in regular exercise at follow-up were asked to identify factors that could motivate them to start an exercise program, and barriers that prevented them from exercising. The most common motivating factors among those who responded $(n=53)$ were improvement in their own health $(n=11$; $21 \%$ of responses), availability of local programs and/or transport to programs $(n=6 ; 11 \%)$, and having more time to take part ( $n=5 ; 9 \%)$. Among those who responded to the question on barriers to exercising $(n=55)$, the most commonly reported were ill health $(n=18 ; 33 \%$ of responses), insufficient time ( $n=11 ; 20 \%)$, and inconvenience of location or time of available exercise classes $(n=8 ; 15 \%)$.

\section{Impact on physical activity and use of safe behaviours}

At follow-up, some domains of weekly physical activity, as reported on the IPEQ, had increased. There was a significant increase in structured exercise from 2 (SD 3.3) hours per week at baseline to 4 (SD 4.7) hours per week at follow-up (mean difference 2.0; 95\% Cl 1.6, 2.5; $p<0.0001$ ), and nonsignificant increases in total physical activity (mean difference 1.1; 95\% Cl 0.9, 3.0; $p=0.3$ ) and incidental physical activity (mean difference 1.2; $95 \% \mathrm{Cl} 0.7,3.1 ; p=0.2)$. At baseline, the average FaB Scale score was 2.9 out of 4 (SD 0.4), and this increased to 3.1 (SD 0.4), at 6-month follow-up; this was a small but statistically significant change (mean difference 0.15 ; 95\% $\mathrm{Cl} 0.12,0.19 ; p<0.0001)$, indicating an increase in safer behaviours at follow-up.

\section{Discussion}

Overall, this study demonstrates that participation in the Stepping On fall prevention program was associated with uptake of two important fall prevention strategies: balance and strength training exercise, and safe walking strategies. Since participation in balance and strength training exercise has previously been shown to be the most effective single intervention for preventing falls in community-dwelling older people, this is a very encouraging result. The results also indicate high acceptability of, and satisfaction with, the Stepping On program, with $98 \%$ of survey respondents willing to recommend the program to others.

These results are in agreement with research involving the Stepping On program delivered to older people in North Dakota, US ${ }^{11}$, which also found high participant satisfaction with the program and a significant impact of the program on knowledge and behaviour regarding fall risk reduction. Research in Wisconsin, US ${ }^{9}$, also demonstrated high stakeholder acceptance of the Stepping On program, which has led to widespread implementation of the program throughout that state.

The Stepping On program focuses on creating positive lifestyle changes to promote independence through both group-based discussion and exercise, and home-based practice of exercises and other protective behaviours. This approach is in accordance with previous research demonstrating that older people prefer fall prevention programs that include social support and interaction, lowintensity exercise, education, and personal relevance. ${ }^{16}$

The strengths of our study include the fact that we surveyed people from all NSW LHDs, providing a mix of participant views and experiences from both urban and regional areas, which suggests that the results are broadly applicable. Furthermore, the proportion of participants reporting chronic health conditions in our sample was comparable to the chronic disease profile reported for the broader Australian population of older 
people. For example, in our sample $16 \%$ of participants reported having diabetes, $14 \%$ reported having asthma and $13 \%$ reported having suffered a stroke, compared with approximately $17 \%{ }^{17}, 11 \%{ }^{17}$ and $12 \%{ }^{18}$ respectively among older Australians.

\section{Limitations}

We acknowledge that this study has certain limitations, and our results need to be considered in light of these limitations. First, we had a large amount of missing data, with only $64 \%$ of participants completing the follow-up survey. This may have been a source of bias, because it is possible that participants who had a more positive view or experience of the Stepping On program may have been more likely to complete the follow-up survey, and this may have overestimated the positive effects and acceptability of the program. Second, the study sample was predominantly female; although this is common for fall prevention research, it may reduce the generalisability of the results to older men. Third, the study relied on selfreported measures of physical activity, and fall prevention behaviours and strategies, which may be subject to recall and reporting bias. Lastly, this study did not measure fall outcomes, so the effect of Stepping On participation on falls in this sample cannot be determined.

\section{Conclusion}

This study provides a snapshot of positive participant views of the Stepping On fall prevention program, and the impact of participation on strategies and behaviours known to prevent falls, such as balance and strength exercises, and safe walking strategies, among adults in the community aged 65 and older. These results demonstrate the appeal of the Stepping On program and its impact on protective fall prevention behaviours.

\section{Acknowledgements}

This work is supported by a National Health and Medical Research Council (NHMRC) Partnerships for Better Health Grant (ID 1016876). AT, CT and SL receive salary funding through NHMRC fellowships. This funder had no role in the study design, execution, analyses, interpretation, or decision to submit results.

\section{Peer review and provenance}

Externally peer reviewed, not commissioned.

\section{Competing interests}

$\mathrm{LC}$ is one of the developers of the Stepping On program.

\section{Author contributions}

CS, AT, SRL and LC conceived the study and initiated the study design, and KP assisted with implementation. AT, CS, SRL and LC are grant holders. AT conducted the primary statistical analysis. All authors contributed to refinement of the study protocol and approved the final manuscript.

\section{References}

1. World Health Organization. WHO global report on falls prevention in older age. Geneva: WHO; 2007 [cited 2019 May 4]. Available from: extranet.who.int/agefriendlyworld/ wp-content/uploads/2014/06/WHo-Global-report-on-fallsprevention-in-older-age.pdf

2. Tinetti ME, Williams CS. Falls, injuries due to falls, and the risk of admission to a nursing home. N Engl J Med. 1997;337(18):1279-84.

3. NSW Government. HealthStats NSW: Prevalence of falls in the elderly. Sydney; NSW Ministry of Health: 2016 [cited 2019 May 4]. Available from: www.healthstats.nsw. gov.au/Indicator/inj_falloldprev_age/inj_falloldprev_age

4. Watson W, Clapperton AJ, Mitchell R. The incidence and cost of falls injury among older people in New South Wales 2006/07. Sydney: NSW Department of Health; 2010 [cited 2019 May 4]. Available from: www.health.nsw. gov.au/falls/Publications/incidence-cost-of-falls.pdf

5. Australian Institute of Health and Welfare. Australian hospital statistics 2010-11. Canberra: AlHW; 2012 [cited 2019 May 4]. Available from: www.aihw.gov.au/reports/ hospitals/australian-hospital-statistics-2010-11/contents/ table-of-contents

6. Sherrington C, Fairhall NJ, Wallbank GK, Tiedemann A Michaleff ZA, Howard K, et al. Exercise for preventing falls in older people living in the community. Cochrane Database of Systematic Reviews. 2019;1:CD012424.

7. Hopewell S, Adedire O, Copsey BJ, Boniface GJ, Sherrington C, Clemson L, et al. Multifactorial and multiple component interventions for preventing falls in older people living in the community. Cochrane Database of Systematic Reviews. 2018;7:CD012221.

8. Clemson L, Cumming RG, Kendig H, Swann M, Heard $\mathrm{R}$, Taylor K. The effectiveness of a community-based program for reducing the incidence of falls in the elderly: a randomized trial. J Am Geriatr Soc. 2004;52(9):148794.

9. Mahoney JE. "Stepping on": stepping over the chasm from research to practice. Front Public Health. 2015;2:148.

10. Ory MG, Smith ML, Jiang L, Lee R, Chen S, Wilson AD, et al. Fall prevention in community settings: results from implementing stepping on in three states. Front Public Health. 2014;2:232. 
11. Strommen J, Brotherson S, Yeng Z. Older adult knowledge and behavior change in the Stepping On fall prevention program in a community setting. J Human Sciences Extension. 2017;5(3):99-121.

12. von Elm E, Altman DG, Egger M, Pocock SJ, Gotzsche PC, Vandenbroucke JP. The Strengthening the Reporting of Observational Studies in Epidemiology (STROBE) statement: guidelines for reporting observational studies. J Clin Epidemiol. 2008;61(4):34449.

13. Clemson L, Bundy AC, Cumming RG, Kay L, Luckett T. Validating the Falls Behavioural (FaB) scale for older people: a Rasch analysis. Disabil Rehabil. 2008;30(7):498-506.

14. Clemson L, Cumming RG, Heard R. The development of an assessment to evaluate behavioral factors associated with falling. Am J Occ Ther. 2003;57(4):380-88.
15. Delbaere K, Hauer K, Lord SR. Evaluation of the incidental and planned exercise questionnaire (IPEQ) for older people. Br J Sport Med. 2010;44(14):1029-34.

16. Bunn F, Dickinson A, Barnett-Page E, Mclnnes E, Horton K. A systematic review of older people's perceptions of facilitators and barriers to participation in falls-prevention interventions. Ageing Society. 2008;28(4):449-72.

17. Australian Bureau of Statistics. National Health Survey: First Results, 2017-18. Canberra: ABS; 2018 [cited 2020 Apr 14]. Available from: www.abs.gov.au/ausstats/abs@. $\mathrm{nst} / \mathrm{mf} / 4364.0 .55 .001$

18. Australian Institute of Health and Welfare 2013. Stroke and its management in Australia: an update. Cardiovascular disease series no. 37. Cat. no. CVD 61 Canberra: AlHW; 2013 [cited 2020 Apr 14]. Available from: www.aihw.gov.au/reports/heart-stroke-vasculardiseases/stroke-management-australia-update/contents/ table-of-contents

\section{Copyright: @) $(1)(0$}

(C) 2020 Tiedemann et al. This article is licensed under the Creative Commons Attribution-NonCommercial-ShareAlike 4.0 International Licence, which allows others to redistribute, adapt and share this work non-commercially provided they attribute the work and any adapted version of it is distributed under the same Creative Commons licence terms. See: www.creativecommons.org/licenses/by-nc-sa/4.0/ 